\title{
Emerging landscape of circHIPK3 and its role in cancer and other diseases (Review)
}

\author{
QI SHAO, YONG HUANG, CAI ZHANG, XIAOCHAN GAO and SHIYANG GAO
}

College of Animal Science and Technology, Henan University of Science and Technology, Luoyang, Henan 471023, P.R. China

Received September 12, 2020; Accepted February 19, 2021

DOI: $10.3892 / \mathrm{mmr} .2021 .12048$

\begin{abstract}
Circular RNAs (circRNAs) are a special class of recently re-discovered RNAs, which are covalently closed ring RNA molecules. circRNAs have been reported to possess multiple functions and are considered crucial regulators of several processes, and are therefore gaining increasing attention. In recent years, increasing evidence has shown that circRNAs are implicated in several crucial biological processes via regulation of gene expression, and their dysregulation is also associated with the development of numerous diseases, particularly acting as oncogenic or tumor-suppressor molecules in cancer. Furthermore, circRNAs are involved in cell proliferation, differentiation, apoptosis, invasion and metastasis. In the present review, the biogenesis and functions of circRNAs are described, with a focus on the most recent research advances and the emerging roles of circular homeodomain-interacting protein kinase 3 (circHIPK3) in human diseases. The present review may provide novel avenues for research on the roles of circHIPK3 as a clinical diagnostic and prognostic biomarker, as well as highlighting promising therapeutic targets for certain diseases and cancer.
\end{abstract}

\section{Contents}

1. Introduction

2. Biogenesis of circRNAs

3. Biological functions of circRNAs

4. circHIPK3 and human diseases

5. Conclusions and future perspectives

Correspondence to: Dr Yong Huang, College of Animal Science and Technology, Henan University of Science and Technology, 263 Kaiyuan Avenue, Luoyang, Henan 471023, P.R. China E-mail: huangyong1979111@163.com

Key words: circular RNA, circular homeodomain-interacting protein kinase 3, diseases, cancer

\section{Introduction}

Circular RNAs (circRNAs) are endogenous single-stranded non-coding RNAs (ncRNAs) generated from protein-coding genes without caps and tails at $5^{\prime}$ and $3^{\prime}$ ends of their structure and shaped as covalently closed continuous loops $(1,2)$. Due to their circular structure, circRNAs are more stable and resistant to degradation by exonuclease RNase R compared with linear mRNAs (3). circRNAs were once considered a product of mis-splicing or splicing noise of precursor mRNAs (pre-mRNAs) (4). circRNAs are now an increasingly popular subject of study in the field of scientific research, particularly in regard to ncRNAs. Moreover, circRNAs are attracting significant attention as functional regulators, and have an important value in biomedical research, particularly with regard to their clinical potential. circRNAs were first discovered in 1976, in a study of RNA viruses (5). Subsequently, Hsu and Coca-Prados (6) first identified the existence of circRNAs in the cytoplasm of eukaryotic cells using electron microscopy in 1979 . In the 21st century, benefiting from the breakthroughs in next generation sequencing technologies and rapid development of bioinformatics, numerous circRNAs have been identified in various organisms (7-9).

Additionally, it has been reported that circRNAs are widely expressed in a variety of living organisms and are evolutionarily conserved amongst different species and often display cell or tissue/developmental-stage-specific expression (10-12). Furthermore, several studies have revealed that circRNAs exhibit aberrant or differential expression in different tissues and disease states, suggesting their important functions in physiological and pathological processes (13-18).

Homeodomain-interacting protein kinase 3 (HIPK3) is a member of the HIPK gene family. circHIPK3 is a circular RNA derived from exon2 of the HIPK3 gene, which is 1,099 nucleotides in length (Fig. 1) (19-21). An increasing number of studies have revealed that circHIPK3 is strongly associated with the occurrence and development of several human diseases, such as osteosarcoma, hepatocellular carcinoma and colorectal cancer $(22,23)$. circHIPK 3 has a regulatory role as a modulator of cellular behavior, as well as exhibiting oncogenic or tumor suppressor functions, depending on the specific diseases $(24,25)$. Thus, circHIPK3 may serve as a novel candidate of diagnosis and prognosis for disease biomarkers, as well as a promising therapeutic molecular target. In the present study, the biogenesis and possible functions of circRNAs are 
discussed, with a focus on recent progress on the study of circHIPK 3 and its association with human diseases and cancer. The aim of the present review was to broaden the knowledge on circHIPK3 and aid future studies assessing the regulatory function of circHIPK3 in the development and progression of diseases.

\section{Biogenesis of circRNAs}

It is generally acknowledged that circRNAs are transcribed from linear pre-mRNAs by RNA polymerase II (RNA Pol II) and formed via back splicing, differing from canonical splicing to form mRNAs (26). According to their genomic origin, circRNAs can be divided into four types: Exonic circRNAs, circular intronic RNAs (ciRNAs), exon-intron circRNAs and tRNA intronic circRNAs (tricRNAs) $(1,27,28)$. Several studies have demonstrated that the biogenesis of circRNAs occurs via four primary models: Lariat-driven circularization path, intron-pairing driven circularization path, RNA-binding protein (RBP)-binding-driven circularization path and intron-splicing driven circularization path (Fig. 2) $(29,30)$ The first model is the lariat-driven circularization path, which requires an upstream 3 ' splice site to be joined to a downstream 5 ' splice site, resulting in exon skipping to form an RNA lariat consisting of several exons and introns (31). Intron-pairing-driven circularization is the second model of circRNA biogenesis. circRNA formation is dependent on ALU elements or flanking inverted repetitive sequences to promote circularization by base-pairing across different introns (32). Notably, ciRNA biogenesis requires a key motif consisting of both a 7-nt GU-rich element near the 5' splice site and an 11-nt C-rich element near the branch point site (33). The third model is called RBP-binding driven circularization, as RBP has an important function in initiating circRNA formation via regulation of adjacent splice site (34). Finally, the fourth model is known as intron-splicing-driven circularization; the pre-tRNA is identified and spliced by the tRNA splicing nuclease complex to remove the excised tRNA introns, which then release and ligate the intron termini to form a tRNA and a tricRNA $(35,36)$.

\section{Biological functions of circRNAs}

To date, the majority of biological functions attributed to circRNAs remain largely incompletely understood. circRNA has a multitude of reported functions, which include serving as a miRNA and protein sponge and transcriptional regulator, and can be translated into protein (Fig. 3).

MicroRNA (miRNA/miR) and protein sponges. circRNAs can bind miRNAs to inhibit their function as competitive endogenous RNAs or miRNA sponges (37). An example of this is circRNA Cdrlas (also known as ciRS-7), which was first reported to function as a sponge of miR-7, containing $>70$ conserved miR-7 binding sites, and thereby significantly decreasing miR-7 levels when its expression is increased $(3,38)$. Similarly, another established example is circSRY, which is derived from the sex-determining region of the $\mathrm{Y}$ chromosome and is composed of 16 binding sites for miR-138 and specifically expressed in the testis (39). Furthermore, numerous studies have demonstrated that circRNAs can function as miRNA sponges. For example, circHIPK3 can regulate cell proliferation by functioning as a sponge of miR-124, inhibiting its expression (20). circNT5E can directly bind miR-422a, acting as a sponge to regulate cell proliferation, migration and invasion in glioblastoma (40). Yang et al (41) has reported that circAmotll can bind with c-myc to induce c-myc nuclear translocation and prevent c-myc mRNA degradation, ultimately promoting tumorigenesis.

Studies have shown that circRNAs can also act as a protein or RBP sponge. For example, circMbl, which is derived from the second exon of muscleblind ( $\mathrm{Mbl})$, contains the binding sites for the Mbl protein; circMb1 is able to directly bind with $\mathrm{Mbl}$ to regulate $\mathrm{Mbl}$ expression by competing with conventional pre-mRNA splicing, thereby decreasing the levels of $\mathrm{Mbl}$ and in turn affecting the formation of circMbl $(26,42)$. The human antigen $\mathrm{R}(\mathrm{HuR})$ protein is an RBP, which is indispensable for promoting the translation of the mRNAs (43).

circPABPN1 has been found to compete with poly(A) binding protein nuclear 1 (PABPN1) mRNA to prevent HuR from binding with PABPN1 mRNA, suppressing its translation (44). circFoxo3, which has recently received increasing attention, has been found to be primarily located in the cytoplasm, where it has been demonstrated to serve as an adaptor bridging p21 and cyclin-dependent kinase 2 (CDK2) (45). Moreover, circFoxo3 can repress cell cycle progression via interaction with p21 and CDK2 to establish a circFoxo3/p21/CDK2 ternary complex inducing cell cycle regulation (45). Additionally, a recent study has revealed that circCcnb1 can interact with both CCNB1 and CDK1 proteins to suppress migration, invasion and proliferation of HTB126 cells (46).

circRNAs as transcriptional regulators. Zhang et al (28) has found that both circRNAs ci-ankrd52 and ci-sirt7 can combine with RNA Pol II in cis, and positively enhance the transcriptional activity of the host genes. Similarly, Li et al (47) has revealed that circEIF3J and circPAIP2 can interact with U1 small nuclear ribonucleic proteins to form a complex, and then bind to Pol II at the promoter region of the host genes to regulate transcription of their host genes. It has been suggested that circRNAs are involved in the regulation of alternative splicing. In 1998, Chao et al (48) reported that the Fmn gene could produce a circRNA via back splicing. In another example, a circRNA from SEPALLATA3 (SEP3) gene regulates splicing of its host SEP3 mRNA via formation of the R-loop, resulting in the suspension of host gene transcription (49). Similar observations have also been attributed to circITCH, which is involved in the regulation of the expression of its host gene (ITCH) indirectly, via sponging its targets miR-1, miR-17 and miR-214 (50).

Translation. As circRNAs lack a 5'-cap and a 3'-end, they were initially shown to not possess protein encoding capacity. However, emerging evidence has indicated that circRNAs may function as a template for translation or synthesis of proteins or peptides (51).

circRNAs were first suggested to possess protein encoding capacity if the sequence contained an internal ribosome entry site (IRES), in a study published in 1995 (52). A subsequent 


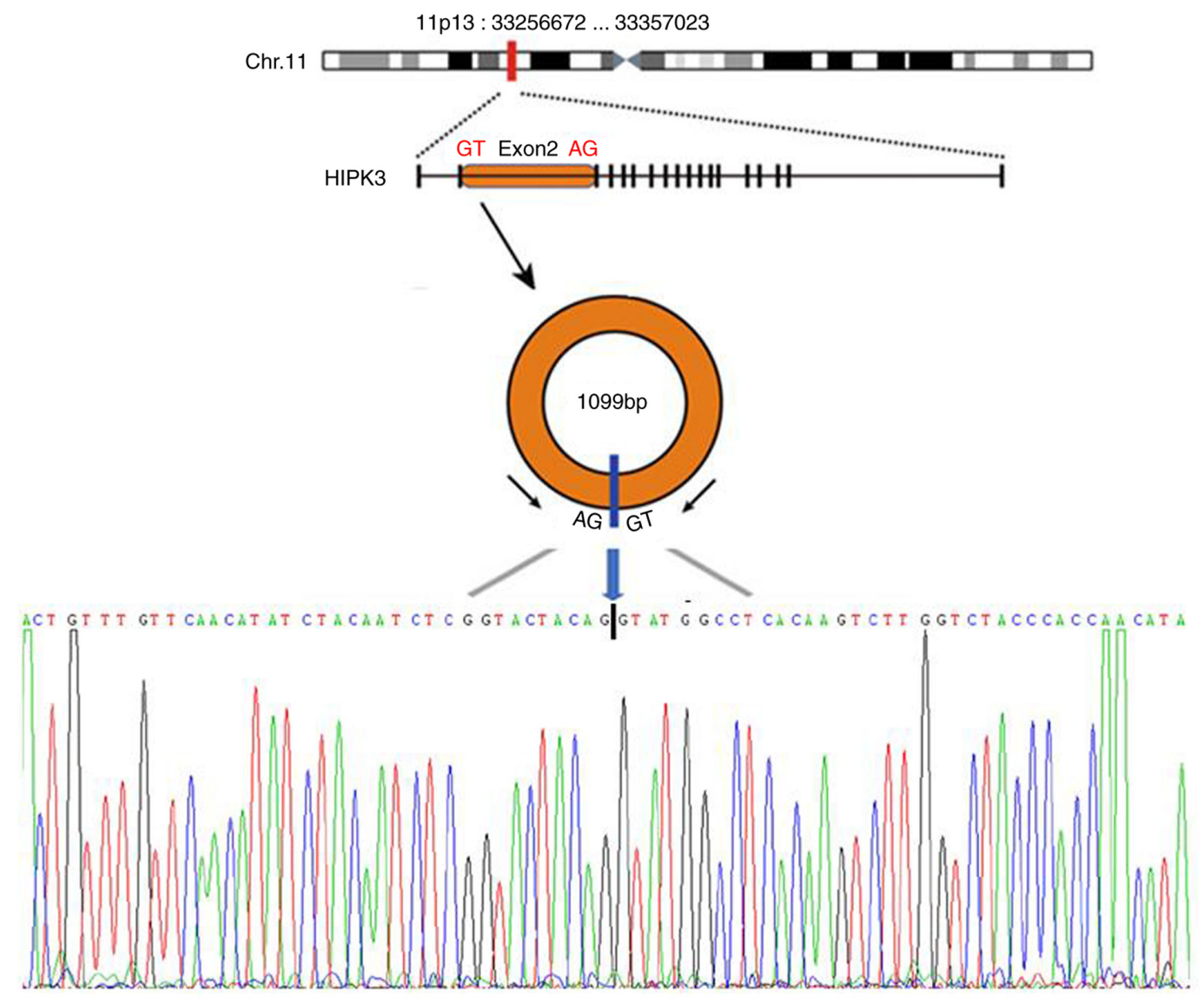

Figure 1. Schematic diagram of generation of circHIPK3. The circularization of HIPK3 exon 2 forms circHIPK3 (black arrow). Bule arrow represents 'head-to-tail' splicing sites of circHIPK3 by Sanger sequencing. circHIPK3, circular homeodomain-interacting protein kinase 3; Chr, chromosome.

study revealed that circZNF609 could translate proteins in murine myoblasts when driven by IRES in a splicing-dependent and cap-independent manner, which indicated that circRNAs exhibited protein-coding capacity (53). Yang et al (54) demonstrated that circFBXW7 could be translated into a novel 21-kDa functional protein [FBXW7-185 amino acids (aa) and its expression was negatively associated with glioblastoma. Another study reported that circSHPRH contained an open reading frame and encoded a 17-kDa protein (SHPRH-146aa) driven by IRES (55). Furthermore, both circSHPRH and SHPRH-146aa expression was decreased in glioblastoma, thereby suppressing cell proliferation and tumorigenesis (55). In addition, a more recent study by Yang et al (56) demonstrated that N-methyladenosine, the most frequent RNA modification, could promote efficient initiation of protein translation from circRNAs. circPINT, which is derived from exon 2 of long intergenic non-protein coding RNA p53-induced transcript (LINC-PINT), can translate proteins, termed PINT87aa encoded by the circular, but not the linear, form of LINC-PINT (57). Whether these translatable circRNAs serve a physiological function remains to be experimentally investigated and confirmed.

\section{4. circHIPK3 and human diseases}

circHIPK3 and prostate cancer $(P C)$. PC is a common malignancy of the urinary system and is a leading cause of cancer-associated death in men (58). Cai et al (59) revealed that
circHIPK3 expression was upregulated in PC tissues and cells. Overexpression of circHIPK 3 accelerated the proliferation and invasiveness of PC cells by sponging miR-338-3p to regulate ADAM17 expression (59). This result provides a potentially novel preventative and therapeutic target for the management of PC. Similarly, Chen et al (60) illustrated that circHIPK3 mediates miR-193a-3p/MCL1 signaling to promote cell proliferation and invasion of PC.

circHIPK3 and lung cancer $(L C)$. LC is a major health threat and the largest cause of cancer-associated death worldwide (61). Studies have revealed that circHIPK3 exerts oncogenic properties in LC. Yu et al (62) reported that circHIPK3 regulated the expression levels of sphingosine kinase 1, CDK4 and STAT3 by acting as a miR-124 sponge in LC. Recently, Lu et al (63) assessed the clinical significance of circHIPK3 in patients with primary non-small cell LC (NSCLC). Further study (63) demonstrated that circHIPK3 induced cell proliferation and inhibited apoptosis in NSCLC by sponging miR-149, indirectly increasing FOXM1 expression. Similarly, Hong et al (64) confirmed that circHIPK3 promoted NSCLC progression via a circHIPK3/miR-107/brain-derived neurotrophic factor (BDNF) axis, highlighting potential markers for NSCLC screening. A study by Chen et al (65) demonstrated that circHIPK3 functioned as an oncogene, and loss of circHIPK3 significantly impaired cell proliferation, migration and invasion, and induced protective autophagy via a miR-124-3p/STAT3/protein kinase AMP-activated catalytic 
A Intron-pairing-driven circularization
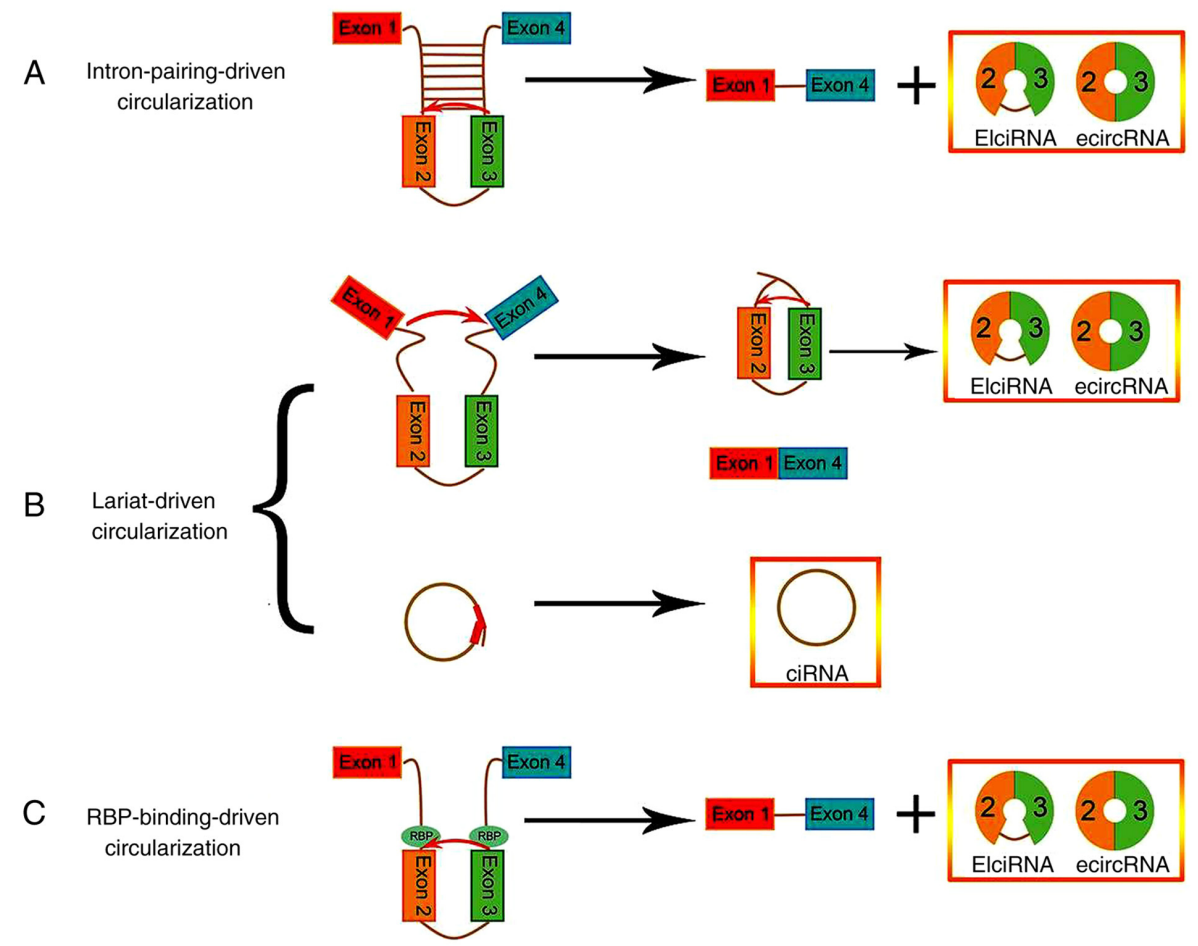

D Intron-splicing-driven cirularization

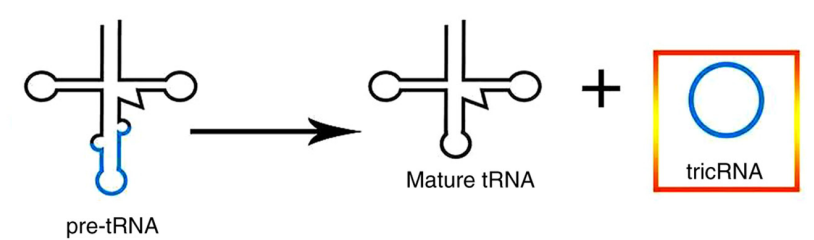

Figure 2. Four main models of circRNA biogenesis. (A) Intron-pairing-driven circularization: ALU elements or flanking inverted repeats form circRNA by intron pairing and the formation of EIciRNAs or ecircRNAs occurs as introns are removed or retained. (B) Lariat-driven circularization. Upstream 3' splice site joins with a downstream 5' splice site, resulting in exon-skipping to form an RNA lariat consisting of multiple exons and introns and leads to formation of EIciRNA, ecircRNA and ciRNA. (C) RBP-binding-driven circularization: RBPs interact with the flanking intronic sequence to form EIciRNA or ecircRNA. (D) Intron-splicing-driven circularization: Pre-tRNAs are synthesized from introns; excised tRNA introns deriving from pre-tRNAs are removed by tRNA splicing enzymes and ligate the released end to form tRNA and tricRNA. This image was originally published in a study by Wen et al (36). circRNA, circular RNA; EIciRNA, exon-intron circRNA; ecircRNA, exonic circRNA; tricRNA, tRNA intronic circRNA; ciRNA, circular intronic RNA; RBP, RNA-binding protein.

A miRNA sponges

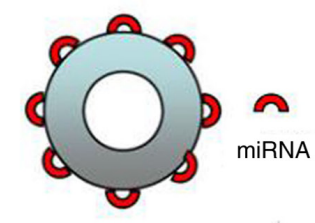

D Competition with pre-mRNA splicing

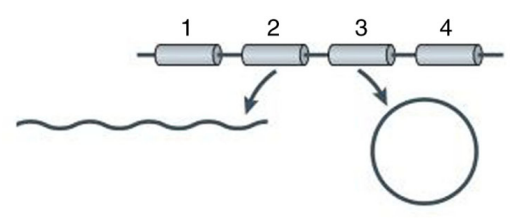

B Protein sponges

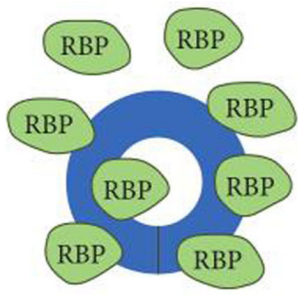

C Transcriptional regulation

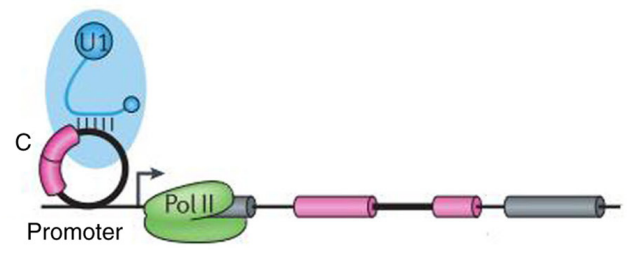

E Templates for translation

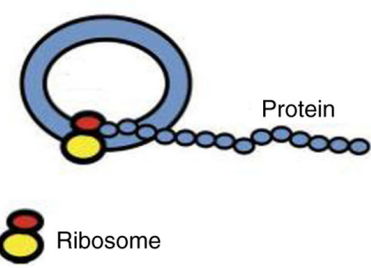

Figure 3. Biological functions of circRNAs. circRNAs can (A) sponge miRNAs, (B) act as protein sponges, (C) regulate host gene transcription by interacting with U1snRNPs and then binding to Pol II, (D) compete with canonical pre-mRNA splicing to facilitate alternative splicing and (E) encode proteins or peptides, acting as templates for translation. circRNA, circular RNA; miRNA, microRNA; RBP, RNA-binding protein; Pol II, polymerase II; U1snRNPs, U1 small nuclear ribonucleoproteins. 

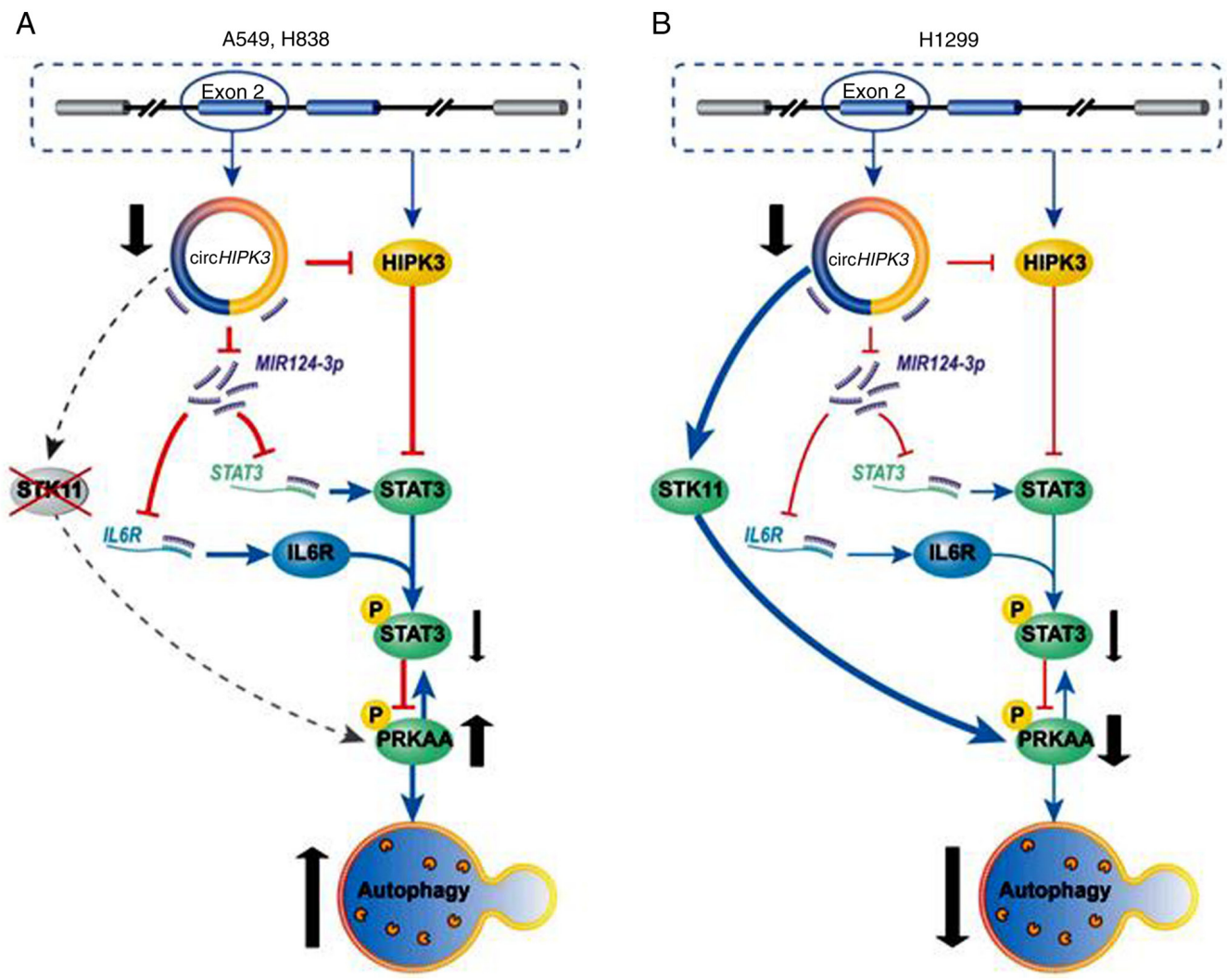

Figure 4. Schematic hypothesis of the regulation of autophagy by circHIPK3. This image was originally published in a study by Chen $e t$ al (65). (A) Lung cancer A549 and H838 cells carried a STK11 mutation and lower STK11 copy number and protein expression to silence circHIPK3-induced autophagy, mainly by decreasing pSTAT3 and increasing pPRKAA signaling. (B) H1299 carried wild-type STK11 to silence circHIPK3-induced autophagy, mainly by decreasing STK11-pPRKAA. p, phosphorylated; STK11, serine/threonine kinase 11; PRKAA, protein kinase AMP-activated catalytic subunit $\alpha 2$; circHIPK3, circular RNA homeodomain-interacting protein kinase 3; MIR, microRNA; IL6R, IL-6 receptor.

subunit a2/AMPKa axis in serine/threonine kinase 11-mutant LC cell lines (A549 and H838; Fig. 4). Overall, the aforementioned results highlight the potential prognostic and therapeutic value of circHIPK3 in LC.

circHIPK3 and colorectal cancer (CRC). CRC is the most common gastrointestinal malignant disease, which ranks third in incidence and second in mortality amongst all types of cancer (66). In 2018, a study by Zeng et al (21) was the first study to reveal an association between circHIPK3 and CRC. circHIPK3 was shown to function as an oncogene, resulting in the proliferation, migration and invasion of CRC cells, whilst decreasing apoptosis (21). Furthermore, circHIPK3 expression was upregulated in CRC by sponging miR-7 to sequester and inhibit miR-7 activity, suggesting that circHIPK3 may have value as a prognostic biomarker in CRC (21). Similarly, another study demonstrated that circPIK3 expression was upregulated in CRC cells and further promoted migration, invasion and proliferation of CRC cells by sponging miR-1207-5p, which directly targeted formin-like 2 (FMNL2) (67). These results suggest that circHIPK3 may modulate a miR-1207-5p/FMNL2 axis, highlighting a potentially novel strategy in the management of CRC.

circHIPK3 and gastric cancer (GC). GC is the leading cause of malignant tumor-associated mortality worldwide and is the most common type of digestive tract cancer, accounting for a third of tumor-associated deaths (68). circHIPK3 has been reported to be involved in GC progression by sponging miR-124 and miR-29b to regulate its target genes collagen type I $\alpha 1$ chain (COL1A1), COL4A1 and CDK6 (69). circHIPK3-knockdown inhibits GC cell proliferation (69). Subsequently, Wei et al (70) confirmed that circHIPK3 was upregulated in GC and associated with clinical stage and grade of GC. Furthermore, circHIPK3-knockdown suppressed the proliferation and migration of GC cells via a circHIPK3/miR-107/BDNF axis. Upregulation of circHIPK3 is associated with a poor prognosis, and thus it may serve as a potential target for GC treatment (70).

circHIPK3 and bladder cancer $(B C)$. $\mathrm{BC}$ is the most common type of tumor of the urinary system and the 9th most frequently diagnosed malignant cancer in the world (71). Recently, Li et al (72) identified thousands of differentially expressed circRNAs between human BC tissues and normal control bladder tissues using RNA sequencing. circHIPK3 is significantly downregulated in BC tissues and cell lines by sponging miR-558 and further suppressing the expression of heparanase. Furthermore, overexpression of circHIPK3 significantly decreased invasion, metastasis and angiogenesis of BC cells, suggesting that circHIPK3 was negatively associated with BC grade (72). circHIPK3 may thus function as a tumor suppressor. Additionally, Xie et al (73) found that circHIPK3 expression was low in BC tissue and its 
overexpression promoted gemcitabine sensitivity in patients with BC.

circHIPK3 and nasopharyngeal carcinoma (NPC). NPC is a common malignant tumor in humans, which occurs in the head region (74). In 2018, Ke et al (75) revealed that circHIPK3 expression was higher in NPC tissues and cell lines compared with in normal tissues and cells. circHIPK3 overexpression enhanced tumor cell proliferation, and when circHIPK3 expression was silenced or depleted, proliferation, migration and invasion were significantly decreased in vitro (75). Thus, circHIPK3 seemed to possess an oncogenic role in NPC. Further analyses revealed that circHIPK3 acted as a sponge to negatively regulate miR-4288 expression, which in turn targeted E74-like ETS transcription factor 3 (ELF3), thereby increasing ELF3 expression (75).

circHIPK3 and gallbladder cancer (GBC). GBC is an aggressive and lethal malignancy of the bile duct, and patients often have a poor prognosis (76). In 2018, Kai et al (77) found that circHIPK3 expression in human GBC cells was higher than that in gallbladder epithelial cells. Apoptosis was induced when circHIPK3 expression was knocked down using a small interfering RNA, which potently inhibited survival and proliferation of established and primary human GBC cells. It was further demonstrated that circHIPK3 acted as a sponge of miR-124, ultimately increasing the expression of miR-124 targets (Rho-associated coiled-coil containing protein kinase 1 and CDK6) and decreasing the activity of miR-124 in GBC cells (77).

circHIPK3 and hepatocellular carcinoma (HCC). HCC is the 5th leading type of cancer and the most lethal type of carcinoma worldwide (78). circHIPK3 is significantly upregulated in $\mathrm{HCC}$, where it promotes cell proliferation, highlighting its role as an oncogene (79). Chen et al (79) demonstrated that circHIPK3 regulated HCC via a circHIPK3/miR124/aquaporin 3 (AQP3) axis. Notably, circHIPK3 was upregulated in $\mathrm{HCC}$ cells and promoted cell proliferation and migration by sponging miR-124 and regulating AQP3 expression (79).

circHIPK3 and osteosarcoma (OS). OS is the most common type of primary bone cancer, and is more likely to occur in children and adolescents (80). A recent study by Xiao-Long et al (81) revealed that circHIPK3 expression was significantly downregulated in OS cell lines and tissues, and its low expression was associated with a poor prognosis. Functional analysis revealed that downregulated circHIPK3 expression resulted in a shorter OS cell survival time, whereas overexpression of circHIPK3 suppressed proliferation, migration and invasion of OS cells (81). These results suggest that circHIPK3 expression levels may negatively regulate cell behavior and thus that circHIPK3 may be used as a biomarker for OS detection.

circHIPK3 and glioma. Glioma is the most malignant tumor of the adult brain (82). Emerging evidence has revealed that circHIPK3 is associated with glioma, where it functions as an oncogene. For example, circHIPK3 accelerates tumor growth in glioma by sponging miR-124-3p, resulting in upregulation of STAT3 expression(83). Jin et al (84) demonstrated that circHIPK3 overexpression significantly promoted the malignant behaviors of glioma cells, increasing proliferation and invasion by sponging miR-654 via a circHIPK3/miR-654/IGF2BP3insulin-like growth factor 2 mRNA binding protein 3 axis, suggesting circHIPK3 may serve as a potential therapeutic target for the treatment of patients with glioma.

circHIPK3 and epithelial ovarian cancer (EOC). OC is the most fatal gynecological cancer amongst women and has a death rate of $60 \%$ in Asia (85). Teng et al (86) investigated the expression profiles of circRNAs between EOC and normal ovarian tissues using RNA sequencing, revealing that circHIPK3 expression was significantly downregulated in EOC. Moreover, silencing of circHIPK3 promoted the proliferation, migration and invasion of $\mathrm{OC}$ cells, suggesting that circHIPK3 may be an important regulator of OC progression, where it exerts a tumor-suppressive function (86).

circHIPK3 and oral squamous cell carcinoma (OSCC). OSCC is one of the top 10 most common types of cancer of the head and neck worldwide (87). Wang et al (88) analyzed circHIPK3 expression and its clinical significance in OSCC, and its association with miR-124 expression. circHIPK 3 expression in OSCC tissues was significantly higher compared with that of the adjacent non-cancerous tissues. Moreover, miR-124 expression in OSCC tissues was significantly lower compared with that in precancerous tissues. Further correlation analysis revealed that circHIPK3 expression negatively regulated miR-124 expression, and silencing of circHIPK3 expression decreased the proliferation of OSCC cells (88). The aforementioned findings suggest that circHIPK3 may contribute to the occurrence and development of OSCC by regulating miR-124 expression.

circHIPK3 and chronic myeloid leukemia (CML). CML is caused by a reciprocal translocation in chromosomes, and accounts for $15 \%$ of reported cases of leukemia (89). Feng et al (90) investigated the expression profile of circHIPK3 in CML, revealing that its expression was significantly upregulated in peripheral blood mononuclear cells and serum samples from CML compared with in healthy normal samples. Further experiments demonstrated that loss-function of circHIPK3 promoted CML progression, indicating that circHIPK3 may serve as a prognostic biomarker (90).

circHIPK3 and age-related cataracts (ARC). ARC is the leading cause of visual impairment and blindness worldwide (91). Recently, Liu et al (92) reported that circHIPK3 regulated human lens epithelial cell (HLEC) function via a circHIPK3/miR-193a/crystallin $\alpha$ A regulatory network. Furthermore, knockdown of circHIPK3 affected the viability, apoptosis and proliferation of HLECs, suggesting a potential role of circHIPK3 in ARC formation (92). These findings highlight a potentially novel targeted method for the prevention and treatment of ARC.

circHIPK3 and preeclampsia. Preeclampsia, a devastating multisystem syndrome, is becoming an increasingly common disease worldwide, and is associated with a high rate of pregnancy-associated morbidity and mortality (93). 
Zhang et al (94) first explored the possible role of dysregulated circHIPK3 expression and its potential contribution to the pathogenesis of preeclampsia. It was revealed that circHIPK3 was significantly downregulated in preeclampsia compared with healthy pregnant controls. circHIPK3 silencing inhibited the migration, invasion, proliferation and tube formation capacities of HTR8/SVneo cells, and circHIPK3 overexpression significantly promoted these capacities, excluding proliferation.

circHIPK3 and pancreatic cancer $(P C a)$. PCa is a malignant tumor of the digestive system with a low probability of incidence (95). circHIPK3 is expressed in pancreatic tissues (96). Liu et al (97) revealed that circHIPK3 expression was upregulated in PCa tissues and was associated with GEM-resistant PCa tissues and cells. Moreover, circHIPK3 exerted its function by enhancing GEM resistance in PCa cells by sponging miR-330-5p, resulting in upregulation of Ras association domain family member 1 , ultimately regulating $\mathrm{PCa}$ cell proliferation, invasion, migration, epithelial-mesenchymal transition (EMT) and apoptosis (97).

circHIPK3 and acute pancreatitis (AP). AP is a dangerous disease with a high mortality rate (98). Wang et al (99) revealed that circHIPK3 expression was closely associated with inflammation in AP. Silencing its expression inhibited the release of IL-1 $\beta$ and TNF- $\alpha$ (99). Additionally, a recent study demonstrated that circHIPK3 expression was upregulated in the serum of patients compared with AP and in caerulein-stimulated AR42J cells (100). Furthermore, it was revealed that circHIPK3 sponged miR-193a-5p to negatively regulate its expression. Gasdermin D (GSDMD) is a target gene of miR-193a-5p, and is a key gene involved in pyroptosis. Thus, silencing miR-193a-5p reversed the effects of GSDMD. These findings suggest that circHIPK3 may promote pyroptosis and inflammation via regulation of the miR-193a-5p/GSDMD axis in AR42J cells (100).

circHIPK3 and cardiac fibrosis $(C F)$. CF is a common pathological process that often results in death (101). Ni et al (102) revealed that circHIPK 3 promoted the proliferation and migration of cardiac fibroblasts in CF. Furthermore, circHIPK3 expression was markedly increased in $\mathrm{CF}$ and heart tissues following treatment with angiotensin II (Ang II). Inhibition of circHIPK3 prevented Ang II-induced CF by sponging miR-29b-3p. The silencing of circHIPK3 effectively reversed miR-29b-3p-induced promotion of CF function and influenced the expression levels of genes targeted by miR-29b-3p $(\alpha$-smooth muscle actin, COL1A1 and COL3A1), suggesting that circHIPK3 may exhibit potential as a targeted therapy for the management of CF (102).

circHIPK3 and allergic rhinitis (AR). AR is the most common allergic disease affecting individuals of various demographics worldwide (103). circHIPK3 and long non-coding RNA (lncGAS5) were shown to promote differentiation of $\mathrm{T}$ helper 2 cells and aggravate AR via modulating their common target miR-495 (104). Moreover, intranasal administration of circHIPK3/lncGAS5 knockdown lentivirus resulted in a decrease in AR symptoms by downregulating GATA binding protein 3 expression, highlighting a potential therapeutic means for AR management (104).

circHIPK3 and renal cancer $(R C)$. $\mathrm{RC}$ is one of the most common malignant tumors of the urinary system, and the incidence of RC is increasing worldwide (105). Recently, Lai et al (106) revealed that circHIPK3 exerted an oncogenic role, and its expression was upregulated in RC tissues and cells. Additionally, it was shown to promote proliferation and migration, and inhibit the apoptosis of RC cells by competitively binding with miR-485-3p, in-turn indirectly increasing the expression levels of Bcl-2, N-cadherin, vimentin and Ki-67 (106). circHIPK3-knockdown inhibited the proliferation, migration and invasion of RC cells (106). These results provide a promising basis for the molecular-targeted therapy of patients with RC. circHIPK3 may serve as a tumor suppressor in RC progression. Li et al (107) found that the overexpression of circHIPK3 significantly inhibited RC cell invasion and migration in vitro, and it repressed proliferation of RC cells in vitro and in vivo. Additionally, another study by Han et al (108) demonstrated that circHIPK3 promoted clear cell RC cell proliferation and migration by altering miR-5083p/CXCL13 signaling, highlighting a potentially novel target for the molecular treatment of clear cell RC.

circHIPK3 and cervical cancer $(C C)$. CC is the most common malignancy in women worldwide with $>400,000$ new cases of CC diagnosed each year (109). Recently, Qian et al (110) investigated the function of circHIPK 3 and its clinical application in CC. circHIPK3 functioned as a sponge of miR-338-3p, resulting in upregulation of hypoxia-inducible factor (HIF)- $1 \alpha$ expression, and thus promoting $\mathrm{CC}$ cell proliferation and EMT, resulting in tumorigenesis (110). miR-338-3p silencing or HIF-1 $\alpha$ overexpression rescued circHIPK3-knockdown-mediated inhibition or induction of apoptosis in CC cells (110). These findings may serve as a basis in the search for a promising treatment strategy by highlighting the role of the circHIPK3/miR-338-3p/HIF-1 $\alpha$ axis in CC.

circHIPK3 and thyroid cancer (TC). TC is one of the most common endocrine malignancies globally, and it originates from follicular or parafollicular thyroid cells (111). Recently, Shu et al (112) investigated the effects of circHIPK3 on the proliferation of TC cells and migration of TC. It was revealed that circHIPK3 acted as an oncogenic circRNA in $\mathrm{TC}$, promoting tumorigenesis and invasiveness of TC by sponging miR-338-3p, in turn upregulating the expression of its target gene RAB23. Knockdown of circHIPK3 significantly decreased the migration, invasion and proliferation of TC cells (112). These results suggest that circHIPK3 may serve as a novel biomarker for the diagnosis and prognosis of TC.

circHIPK3 and breast cancer (BRC). BRC is one of the most common malignant types of cancer amongst women and is the leading cause of cancer-associated death worldwide (113). $\mathrm{BRC}$ is a major disease threatening female health, affecting $>7 \%$ of women in $>100$ countries (114). Chen et al (115) revealed that circHIPK3 expression was upregulated in BRC, where it facilitated cell proliferation, migration and invasion by targeting miR-193a. Furthermore, overexpression of 


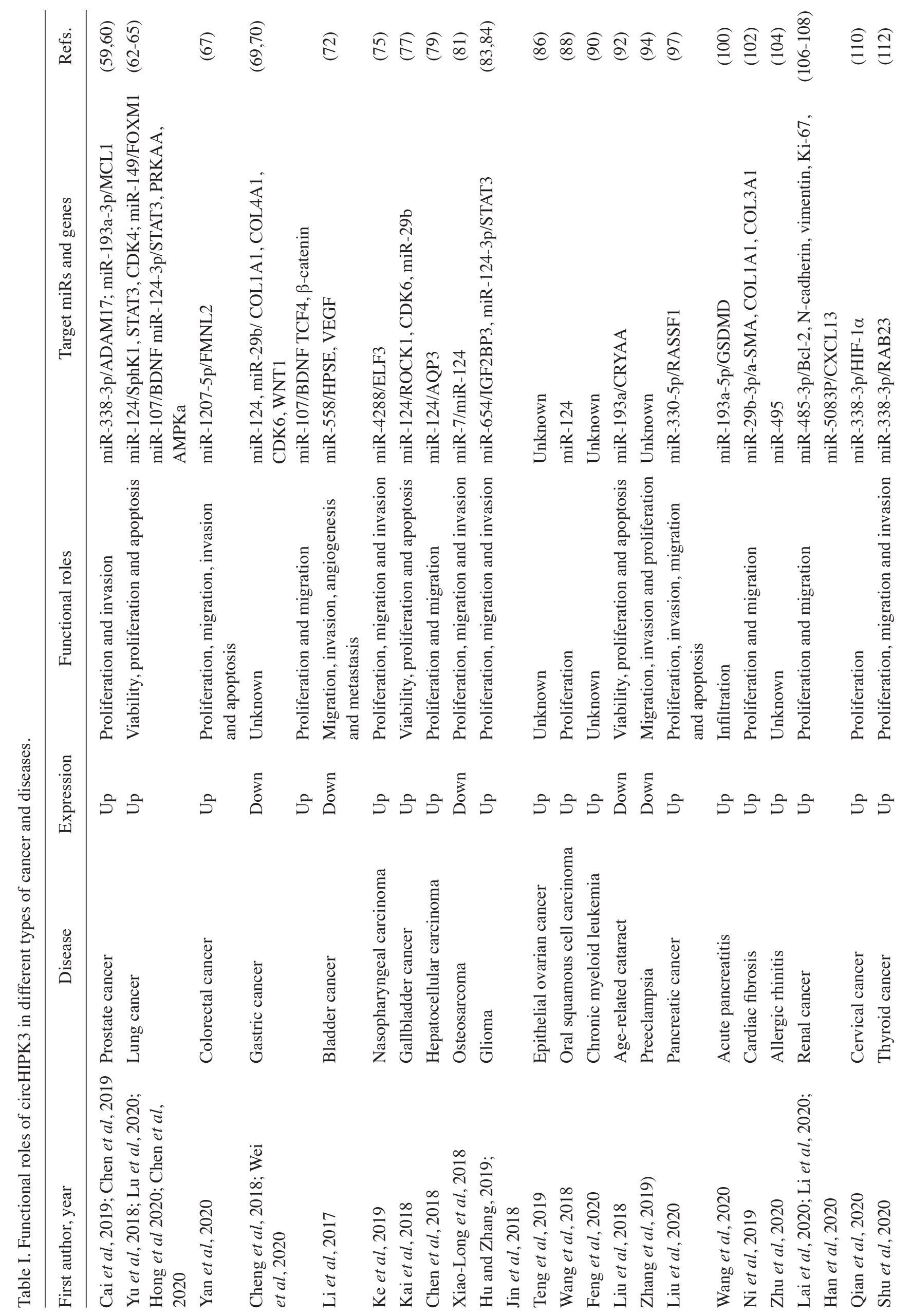


circHIPK3 enhanced high mobility group box 1/PI3K/AKT signaling, highlighting circHIPK3 as a novel potential therapeutic target for BRC management.

\section{Conclusions and future perspectives}

circRNAs have attracted increasing attention over the last decade. In recent years, with the advancement of high throughput RNA sequencing technologies and the rapid development of bioinformatics tools, a large number of circRNAs have been discovered and identified in various organisms. With the efforts of scientists and the application of novel biological methods, the potential role of circRNAs in biological functions is being elucidated. Given the large number of different circRNAs and their potential tissue-specific functions, an in-depth understanding of the complex networks in which they participate and regulate should be developed.

circHIPK3 has been reported to be involved in various human diseases, including different types of cancer. Dysregulation of circHIPK 3 has been observed in a range of diseases and cancerous tissues, where it has been shown to exhibit notable effects on cell cycle progression, cell proliferation, apoptosis, invasion and migration in cancer, suggesting that circHIPK 3 may possess significant value as a molecular biomarker for the diagnosis, prognosis and monitoring of diseases. The vital physiological and pathological functions of circHIPK3 in diseases are listed in Table I. circHIPK3 has been described as both a tumor suppressor and an oncogene. Currently, due to the technical limitations of available methods, the role of circHIPK3 in cancer and various diseases remains to be further elucidated. miRNA sponging is one of the primary mechanisms by which circHIPK 3 exerts its different functions in various diseases. Thus, further studies are required to extensively explore the interaction network of circHIPK 3 in cancer and diseases, including the involvement of miRNAs, lncRNAs, mRNAs and protein degrading pathways. Whether there are other regulatory mechanisms or if they possess other functions should be further studied.

The exact mechanisms by which circHIPK 3 regulates pathological processes remain unclear in certain diseases, such as EOC, CML, AR and preeclampsia. Future studies should explore any other potential mechanisms and functions of circHIPK3. To the best of our knowledge, there is no preclinical evidence for the application of circHIPK 3 as a target or molecular targeted therapeutic tool for cancer treatment. Based on the findings described in the present review, it may be suggested that circHIPK 3 may serve as a predictive biomarker and therapeutic target with clinical promise in the future.

\section{Acknowledgements}

Not applicable.

\section{Funding}

The present review was partly supported by the Natural Science Foundation of China (grant nos. 1471971, 31402263 and 31872537). 


\section{Availability of data and materials}

Not applicable.

\section{Authors' contributions}

QS conceptualized the review, performed the literature search and drafted the manuscript. YH and $\mathrm{CZ}$ helped to draft and revise the manuscript. $\mathrm{XG}$ and $\mathrm{SG}$ edited and revised the paper. All authors have read and approved the final version of the manuscript to be published.

\section{Ethics approval and consent to participate}

Not applicable.

\section{Patient consent for publication}

Not applicable.

\section{Competing interests}

The authors declare that they have no competing interests.

\section{References}

1. Liu J, Liu T, Wang X and He A: Circles reshaping the RNA world: From waste to treasure. Mol Cancer 16: 58, 2017.

2. Jeck WR and Sharpless NE: Detecting and characterizing circular RNAs. Nat Biotechnol 32: 453-461, 2014.

3. Memczak S, Jens M, Elefsinioti A, Torti F, Krueger J, Rybak A, Maier L, Mackowiak SD, Gregersen LH, Munschauer M, et al Circular RNAs are a large class of animal RNAs with regulatory potency. Nature 495: 333-338, 2013.

4. Cocquerelle C, Mascrez B, Hétuin D and Bailleul B: Mis-splicing yields circular RNA molecules. FASEB J 7 : 155-160, 1993

5. Sanger HL, Klotz G, Riesner D, Gross HJ and Kleinschmidt AK Viroids are single-stranded covalently closed circular RNA molecules existing as highly base-paired rod-like structures. Proc Natl Acad Sci USA 73: 3852-3856, 1976.

6. Hsu MT and Coca-Prados M: Electron microscopic evidence for the circular form of RNA in the cytoplasm of eukaryotic cells. Nature 280: 339-340, 1979.

7. Zhang W, Qin P, Gong X, Huang L, Wang C, Chen G, Chen J, Wang $\mathrm{L}$ and Lv Z: Identification of circRNAs in the liver of whitespotted bamboo shark (Chiloscyllium plagiosum). Front Genet 11: 596308, 2020.

8. Zhang J, Liu R, Zhu Y, Gong J, Yin S, Sun P, Feng H, Wang Q, Zhao S, Wang Z and Li G: Identification and characterization of circRNAs responsive to methyl jasmonate in Arabidopsis thaliana. Int J Mol Sci 21: 792, 2020.

9. Li L, Sun D, Li X, Yang B and Zhang W: Identification of key circRNAs in non-small cell lung cancer. Am J Med Sci 361 98-105, 2021

10. Chen LL and Yang L: Regulation of circRNA biogenesis. RNA Biol 12: 381-388, 2015.

11. Geng X, Jia Y, Zhang Y, Shi L, Li Q, Zang A and Wang H: Circular RNA: Biogenesis, degradation, functions and potential roles in mediating resistance to anticarcinogens. Epigenomics 12: 267-283, 2020 .

12. Salzman J: Circular RNA expression: Its potential regulation and function. Trends Genet 32: 309-316, 2016.

13. Wan B, Liu B and Lv C: Progress of research into circular RNAs in urinary neoplasms. PeerJ 8: e8666, 2020.

14. Jahani S, Nazeri E, Majidzadeh-A K, Jahani M and Esmaeili R: Circular RNA; a new biomarker for breast cancer: A systematic review. J Cell Physiol 235: 5501-5510, 2020.

15. Huang G, Li S, Yang N, Zou Y, Zheng D and Xiao T: Recent progress in circular RNAs in human cancers. Cancer Lett 404: 8-18, 2017.
16. Lee ECS, Elhassan SAM, Lim GPL, Kok WH, Tan SW, Leong EN, Tan SH, Chan EWL, Bhattamisra SK, Rajendran R and Candasamy M: The roles of circular RNAs in human development and diseases. Biomed Pharmacother 111: 198-208, 2019.

17. Lei K, Bai H, Wei Z, Xie C, Wang J, Li J and Chen Q: The mechanism and function of circular RNAs in human diseases. Exp Cell Res 368: 147-158, 2018.

18. Soghli N, Qujeq D, Yousefi T and Soghli N: The regulatory functions of circular RNAs in osteosarcoma. Genomics 112: 2845-2856, 2020.

19. Conte A and Pierantoni GM: Update on the regulation of HIPK1, HIPK 2 and HIPK 3 protein kinases by microRNAs. Microrna 7: 178-186, 2018.

20. Zheng Q, Bao C, Guo W, Li S, Chen J, Chen B, Luo Y, Lyu D, Li Y, Shi G, et al: Circular RNA profiling reveals an abundant circHIPK3 that regulates cell growth by sponging multiple miRNAs. Nat Commun 7: 11215, 2016.

21. Zeng K, Chen X, Xu M, Liu X, Hu X, Xu T, Sun H, Pan Y, He B and Wang S: circHIPK3 promotes colorectal cancer growth and metastasis by sponging miR-7. Cell Death Dis 9: 417, 2018.

22. Wen Y, Li B, He M, Teng S, Sun Y and Wang G: circHIPK3 promotes proliferation and migration and invasion via regulation of miR-637/HDAC4 signaling in osteosarcoma cells. Oncol Rep 45: 169-179, 2021.

23. Zhang Y, Liu Q and Liao Q: circHIPK3: A promising cancer-related circular RNA. Am J Transl Res 12: 6694-6704, 2020.

24. Wang J, Zhu M, Pan J, Chen C, Xia S and Song Y: Circular RNAs: A rising star in respiratory diseases. Respir Res 20: 3 , 2019.

25. Xie Y, Yuan X, Zhou W, Kosiba AA, Shi H, Gu J and Qin Z: The circular RNA HIPK3 (circHIPK3) and its regulation in cancer progression: Review. Life Sci 254: 117252, 2020.

26. Ashwal-Fluss R, Meyer M, Pamudurti NR, Ivanov A, Bartok O, Hanan M, Evantal N, Memczak S, Rajewsky N and Kadener S: circRNA biogenesis competes with pre-mRNA splicing. Mol Cell 56: 55-66, 2014.

27. Ragan C, Goodall GJ, Shirokikh NE and Preiss T: Insights into the biogenesis and potential functions of exonic circular RNA. Sci Rep 9: 2048, 2019.

28. Zhang C, Ma L, Niu Y, Wang Z, Xu X, Li Y and Yu Y: Circular RNA in lung cancer research: Biogenesis, functions, and roles. Int J Biol Sci 16: 803-814, 2020.

29. Panda AC, Grammatikakis I, Munk R, Gorospe M and Abdelmohsen K: Emerging roles and context of circular RNAs. Wiley Interdiscip Rev RNA 8: 10.1002/wrna.1386, 2017.

30. Zang J, Lu D and Xu A: The interaction of circRNAs and RNA binding proteins: An important part of circRNA maintenance and function. J Neurosci Res 98: 87-97, 2020.

31. Kelly S, Greenman C, Cook PR and Papantonis A: Exon skipping is correlated with exon circularization. J Mol Biol 427: 2414-2417, 2015.

32. Kristensen LS, Andersen MS, Stagsted LVW, Ebbesen KK, Hansen TB and Kjems J: The biogenesis, biology and characterization of circular RNAs. Nat Rev Genet 20: 675-691, 2019.

33. Zhang Y, Zhang XO, Chen T, Xiang JF, Yin QF, Xing YH, Zhu S, Yang L and Chen LL: Circular intronic long noncoding RNAs. Mol Cell 51: 792-806, 2013.

34. Stagsted LVW, O'Leary ET, Ebbesen KK and Hansen TB: The RNA-binding protein SFPQ preserves long-intron splicing and regulates circRNA biogenesis in mammals. Elife 10: e63088, 2021.

35. Schmidt CA, Giusto JD, Bao A, Hopper AK and Matera AG: Molecular determinants of metazoan tricRNA biogenesis. Nucleic Acids Res 47: 6452-6465, 2019.

36. Wen J, Liao J, Liang J, Chen XP, Zhang B and Chu L: Circular RNA HIPK3: A key circular RNA in a variety of human cancers. Front Oncol 10: 773, 2020.

37. Sumazin P, Yang X, Chiu HS, Chung WJ, Iyer A, Llobet-Navas D, Rajbhandari P, Bansal M, Guarnieri P, Silva J and Califano A: An extensive microRNA-mediated network of RNA-RNA interactions regulates established oncogenic pathways in glioblastoma. Cell 147: 370-381, 2011

38. Hansen TB, Jensen TI, Clausen BH, Bramsen JB, Finsen B, Damgaard CK and Kjems J: Natural RNA circles function as efficient microRNA sponges. Nature 495: 384-388, 2013.

39. Capel B, Swain A, Nicolis S, Hacker A, Walter M, Koopman P, Goodfellow P and Lovell-Badge R: Circular transcripts of the testis-determining gene Sry in adult mouse testis. Cell 73: 1019-1030, 1993. 
40. Wang R, Zhang S, Chen X, Li N, Li J, Jia R, Pan Y and Liang H: CircNT5E acts as a sponge of miR-422a to promote glioblastoma tumorigenesis. Cancer Res 78: 4812-4825, 2018.

41. Yang Q, Du WW, Wu N, Yang W, Awan FM, Fang L, Ma J, Li X Zeng Y, Yang Z, et al: A circular RNA promotes tumorigenesis by inducing c-myc nuclear translocation. Cell Death Differ 24 1609-1620, 2017.

42. Barrett SP and Salzman J: Circular RNAs: Analysis, expression and potential functions. Development 143: 1838-1847, 2016.

43. Joassard OR, Bélanger G, Karmouch J, Lunde JA, Shukla AH, Chopard A, Legay C and Jasmin BJ: HuR mediates changes in the stability of AChR $\beta$-subunit mRNAs after skeletal muscle denervation. J Neurosci 35: 10949-10962, 2015.

44. Abdelmohsen K, Panda AC, Munk R, Grammatikakis I, Dudekula DB, De S, Kim J, Noh JH, Kim KM, Martindale JL and Gorospe M: Identification of HuR target circular RNAs uncovers suppression of PABPN1 translation by circPABPN1. RNA Biol 14: 361-369, 2017.

45. Du WW, Yang W, Liu E, Yang Z, Dhaliwal P and Yang BB: Foxo3 circular RNA retards cell cycle progression via forming ternary complexes with p21 and CDK2. Nucleic Acids Res 44: 2846-2858, 2016.

46. Fang L, Du WW, Awan FM, Dong J and Yang BB: The circular RNA circ-Ccnb1 dissociates Ccnb1/Cdk1 complex suppressing cell invasion and tumorigenesis. Cancer Lett 459: 216-226, 2019.

47. Li Z, Huang C, Bao C, Chen L, Lin M, Wang X, Zhong G, Yu B, $\mathrm{Hu}$ W, Dai L, et al: Exon-intron circular RNAs regulate transcription in the nucleus. Nat Struct Mol Biol 22: 256-264, 2015.

48. Chao CW, Chan DC, Kuo A and Leder P: The mouse formin (Fmn) gene: Abundant circular RNA transcripts and gene-targeted deletion analysis. Mol Med 4: 614-628, 1998

49. Conn VM, Hugouvieux V, Nayak A, Conos SA, Capovilla G Cildir G, Jourdain A, Tergaonkar V, Schmid M, Zubieta C and Conn SJ: A circRNA from SEPALLATA3 regulates splicing of its cognate mRNA through R-loop formation. Nat Plants 3 : 17053, 2017.

50. Li Y, Ge YZ, Xu L and Jia R: Circular RNA ITCH: A novel tumor suppressor in multiple cancers. Life Sci 254: 117176, 2020.

51. Pamudurti NR, Bartok O, Jens M, Ashwal-Fluss R, Stottmeister C, Ruhe L, Hanan M, Wyler E, Perez-Hernandez D, Ramberger E, et al: Translation of CircRNAs. Mol Cell 66: 9-21. e7, 2017.

52. Chen CY and Sarnow P: Initiation of protein synthesis by the eukaryotic translational apparatus on circular RNAs Science 268: 415-417, 1995

53. Legnini I, Di Timoteo G, Rossi F, Morlando M, Briganti F, Sthandier O, Fatica A, Santini T, Andronache A, Wade M, et al: Circ-ZNF609 is a circular RNA that can be translated and functions in myogenesis. Mol Cell 66: 22-37.e9, 2017.

54. Yang Y, Gao X, Zhang M, Yan S, Sun C, Xiao F, Huang N, Yang X, Zhao K, Zhou H, et al: Novel role of FBXW7 circular RNA in repressing glioma tumorigenesis. J Natl Cancer Inst 110: 304-315, 2018

55. Zhang M, Huang N, Yang X, Luo J, Yan S, Xiao F, Chen W, Gao X, Zhao K, Zhou H, et al: A novel protein encoded by the circular form of the SHPRH gene suppresses glioma tumorigenesis. Oncogene 37: 1805-1814, 2018.

56. Yang Y, Fan X, Mao M, Song X, Wu P, Zhang Y, Jin Y, Yang Y, Chen LL, Wang Y, et al: Extensive translation of circular RNAs driven by $\mathrm{N}^{6}$-methyladenosine. Cell Res 27: 626-641, 2017.

57. Zhang M, Zhao K, Xu X, Yang Y, Yan S, Wei P, Liu H, Xu J, Xiao F, Zhou $\mathrm{H}$, et al: A peptide encoded by circular form of LINC-PINT suppresses oncogenic transcriptional elongation in glioblastoma. Nat Commun 9: 4475, 2018.

58. Paschalis A and de Bono JS: Prostate cancer 2020: 'The times they are a'changing'. Cancer Cell 38: 25-27, 2020.

59. Cai C, Zhi Y, Wang K, Zhang P, Ji Z, Xie C and Sun F: circHIPK3 overexpression accelerates the proliferation and invasion of prostate cancer cells through regulating miRNA-338-3p. Onco Targets Ther 12: 3363-3372, 2019.

60. Chen D, Lu X, Yang F and Xing N: Circular RNA circHIPK3 promotes cell proliferation and invasion of prostate cancer by sponging miR-193a-3p and regulating MCL1 expression. Cancer Manag Res 11: 1415-1423, 2019.

61. Siegel RL, Miller KD and Jemal A: Cancer statistics, 2018. CA Cancer J Clin 68: 7-30, 2018.

62. Yu H, Chen Y and Jiang P: Circular RNA HIPK3 exerts oncogenic properties through suppression of miR-124 in lung cancer. Biochem Biophys Res Commun 506: 455-462, 2018.
63. Lu H, Han X, Ren J, Ren K, Li Z and Sun Z: Circular RNA HIPK3 induces cell proliferation and inhibits apoptosis in non-small cell lung cancer through sponging miR-149. Cancer Biol Ther 21: $113-121,2020$

64. Hong W, Zhang Y, Ding J, Yang Q, Xie H and Gao X: circHIPK3 acts as competing endogenous RNA and promotes non-small-cell lung cancer progression through the miR-107/BDNF signaling pathway. Biomed Res Int 2020: 6075902, 2020.

65. Chen X, Mao R, Su W, Yang X, Geng Q, Guo C, Wang Z, Wang J, Kresty LA, Beer DG, et al: Circular RNA circHIPK 3 modulates autophagy via MIR124-3p-STAT3-PRKAA/AMPK $\alpha$ signaling in STK11 mutant lung cancer. Autophagy 16: 659-671, 2020.

66. Malvezzi M, Carioli G, Bertuccio P, Boffetta P, Levi F, La Vecchia C and Negri E: European cancer mortality predictions for the year 2018 with focus on colorectal cancer. Ann Oncol 29: 1016-1022, 2018.

67. Yan Y, Su M and Qin B: circHIPK3 promotes colorectal cancer cells proliferation and metastasis via modulating of miR-1207-5p/FMNL2 signal. Biochem Biophys Res Commun 524: 839-846, 2020.

68. Ilson DH: Advances in the treatment of gastric cancer: 2019. Curr Opin Gastroenterol 35: 551-554, 2019.

69. Cheng J, Zhuo H, Xu M, Wang L, Xu H, Peng J, Hou J, Lin L and Cai J: Regulatory network of circRNA-miRNA-mRNA contributes to the histological classification and disease progression in gastric cancer. J Transl Med 16: 216, 2018.

70. Wei J, Xu H, Wei W, Wang Z, Zhang Q, De W and Shu Y: circHIPK3 promotes cell proliferation and migration of gastric cancer by sponging miR-107 and regulating BDNF expression. Onco Targets Ther 13: 1613-1624, 2020.

71. Siegel RL, Miller KD and Jemal A: Cancer statistics, 2019. CA Cancer J Clin 69: 7-34, 2019.

72. Li Y, Zheng F, Xiao X, Xie F, Tao D, Huang C, Liu D, Wang M, Wang L, Zeng F and Jiang G: circHIPK3 sponges miR-558 to suppress heparanase expression in bladder cancer cells. EMBO Rep 18: 1646-1659, 2017.

73. Xie F, Zhao N, Zhang H and Xie D: Circular RNA circHIPK3 promotes gemcitabine sensitivity in bladder cancer. J Cancer 11: 1907-1912, 2020

74. Akcay M,Etiz D, Celik O and Ozen A: Evaluation of prognosis in nasopharyngeal cancer using machine learning. Technol Cancer Res Treat 19: 1533033820909829, 2020.

75. Ke Z, Xie F, Zheng C and Chen D: circHIPK3 promotes proliferation and invasion in nasopharyngeal carcinoma by abrogating miR-4288-induced ELF3 inhibition. J Cell Physiol 234: 1699-1706, 2019.

76. Sharma A, Sharma KL, Gupta A, Yadav A and Kumar A: Gallbladder cancer epidemiology, pathogenesis and molecular genetics: Recent update. World J Gastroenterol 23: 3978-3998, 2017.

77. Kai D, Yannian L, Yitian C, Dinghao G, Xin Z and Wu J: Circular RNA HIPK 3 promotes gallbladder cancer cell growth by sponging microRNA-124. Biochem Biophys Res Commun 503: 863-869, 2018

78. Torre LA, Bray F, Siegel RL, Ferlay J, Lortet-Tieulent J and Jemal A: Global cancer statistics, 2012. CA Cancer J Clin 65: 87-108, 2015.

79. Chen G, Shi Y, Liu M and Sun J: circHIPK3 regulates cell proliferation and migration by sponging miR-124 and regulating AQP3 expression in hepatocellular carcinoma. Cell Death Dis 9: 175,2018 .

80. Wang Y, Zhang Y, Yang T, Zhao W, Wang N, Li P, Zeng X and Zhang W: Long non-coding RNA MALAT1 for promoting metastasis and proliferation by acting as a ceRNA of miR-144-3p in osteosarcoma cells. Oncotarget 8: 59417-59434, 2017.

81. Xiao-Long M, Kun-Peng Z and Chun-Lin Z: Circular RNA circ_HIPK3 is down-regulated and suppresses cell proliferation, migration and invasion in osteosarcoma. J Cancer 9: 1856-1862, 2018.

82. Quartuccio N, Laudicella R, Vento A, Pignata S, Mattoli MV Filice R, Comis AD, Arnone A, Baldari S, Cabria M and Cistaro A: The additional value of ${ }^{18} \mathrm{~F}-\mathrm{FDG}$ PET and MRI in patients with glioma: A review of the literature from 2015 to 2020. Diagnostics (Basel) 10: 357, 2020

83. Hu D and Zhang Y: Circular RNA HIPK3 promotes glioma progression by binding to miR-124-3p. Gene 690: 81-89, 2019.

84. Jin P, Huang Y, Zhu P, Zou Y, Shao T and Wang O: CircRNA circHIPK3 serves as a prognostic marker to promote glioma progression by regulating miR-654/IGF2BP3 signaling. Biochem Biophys Res Commun 503: 1570-1574, 2018. 
85. Cai Z and Liu Q: Understanding the global cancer statistics 2018: Implications for cancer control. Sci China Life Sci: Aug 26, 2019 (Epub ahead of print). doi: 10.1007/s11427-019-9816-1.

86. Teng F, Xu J, Zhang M, Liu S, Gu Y, Zhang M, Wang X, Ni J, Qian B, Shen R and Jia X: Comprehensive circular RNA expression profiles and the tumor-suppressive function of circHIPK3 in ovarian cancer. Int J Biochem Cell Biol 112: 8-17, 2019.

87. Liang S, Zhang S, Wang P, Yang C, Shang C, Yang J and Wang J: LncRNA, TUG1 regulates the oral squamous cell carcinoma progression possibly via interacting with Wnt/ $\beta$-catenin signaling. Gene 608: 49-57, 2017.

88. Wang J, Zhao SY, Ouyang SS, Huang ZK, Luo Q and Liao L: Circular RNA circHIPK3 acts as the sponge of microRNA-124 to promote human oral squamous cell carcinoma cells proliferation. Zhonghua Kou Qiang Yi Xue Za Zhi 53: 546-551, 2018 (In Chinese).

89. Minciacchi VR, Kumar R and Krause DS: Chronic myeloid leukemia: A model disease of the past, present and future. Cells 10: 117, 2021

90. Feng XQ, Nie SM, Huang JX, Li TL, Zhou JJ, Wang W, Zhuang LK and Meng FJ: Circular RNA circHIPK3 serves as a prognostic marker to promote chronic myeloid leukemia progression. Neoplasma 67: 171-177, 2020.

91. Congdon NG, Friedman DS and Lietman T: Important causes of visual impairment in the world today. JAMA 290: 2057-2060, 2003

92.Liu X, Liu B, Zhou M, Fan F, Yu M, Gao C, Lu Y and Luo Y: Circular RNA HIPK3 regulates human lens epithelial cells proliferation and apoptosis by targeting the miR-193a/CRYAA axis. Biochem Biophys Res Commun 503: 2277-2285, 2018.

93. Gathiram P and Moodley J: Pre-eclampsia: Its pathogenesis and pathophysiolgy. Cardiovasc J Afr 27: 71-78, 2016.

94.Zhang Y, Cao L, Jia J, Ye L, Wang Y, Zhou B and Zhou R: circHIPK3 is decreased in preeclampsia and affects migration, invasion, proliferation, and tube formation of human trophoblast cells. Placenta 85: 1-8, 2019.

95. Vera R, Dotor E, Feliu J, González E, Laquente B, Macarulla T, Martínez E, Maurel J, Salgado M and Manzano JL: SEOM clinical guideline for the treatment of pancreatic cancer (2016). Clin Transl Oncol 18: 1172-1178, 2016.

96. Xu T, Wu J, Han P, Zhao Z and Song X: Circular RNA expression profiles and features in human tissues: A study using RNA-seq data. BMC Genomics 18 (Suppl 6): S680, 2017.

97. Liu Y, Xia L, Dong L, Wang J, Xiao Q, Yu X and Zhu H: circHIPK 3 promotes gemcitabine (GEM) resistance in pancreatic cancer cells by sponging miR-330-5p and targets RASSF1. Cancer Manag Res 12: 921-929, 2020.

98. Rahman A, O'Connor DB, Gather F, Koscic S, Gilgan J, Mockler D, Bashir Y, Memba R, Duggan SN and Conlon KC: Clinical classification and severity scoring systems in chronic pancreatitis: A systematic review. Dig Surg 37: 181-191, 2020.

99. Wang L, Luo T, Bao Z, Li Y and Bu W: Intrathecal circHIPK3 shRNA alleviates neuropathic pain in diabetic rats. Biochem Biophys Res Commun 505: 644-650, 2018.

100. Wang J, Li X, Liu Y, Peng C, Zhu H, Tu G, Yu X and Li Z: circHIPK3 promotes pyroptosis in acinar cells through regulation of the miR-193a-5p/GSDMD axis. Front Med (Lausanne) 7: $88,2020$.

101. Holmström L, Haukilahti A, Vähätalo J, Kenttä T, Appel H, Kiviniemi A, Pakanen L, Huikuri HV, Myerburg RJ and Junttila J. Electrocardiographic associations with myocardial fibrosis among sudden cardiac death victims. Heart 106: 1001-1006, 2020
102. Ni H, Li W, Zhuge Y, Xu S, Wang Y, Chen Y, Shen G and Wang F: Inhibition of circHIPK3 prevents angiotensin II-induced cardiac fibrosis by sponging miR-29b-3p. Int J Cardiol 292: 188-196, 2019.

103. Gotua M, Gamkrelidze A, Rukhadze M, Abramidze T, Bochorishvili E, Shengelidze G, Dolidze N, Chkhartishvili E, Bachert C, Pfaar O, et al: 2020 Aria care pathways for allergic rhinitis-georgia. Georgian Med News: 108-117, 2019.

104. Zhu X, Wang X, Wang Y and Zhao Y: The regulatory network among circHIPK3, LncGAS5, and miR-495 promotes Th2 differentiation in allergic rhinitis. Cell Death Dis 11: 216, 2020.

105. Taneja K and Williamson SR: Updates in pathologic staging and histologic grading of renal cell carcinoma. Surg Pathol Clin 11: 797-812, 2018

106. Lai J, Xin J, Fu C and Zhang W: circHIPK3 promotes proliferation and metastasis and inhibits apoptosis of renal cancer cells by inhibiting MiR-485-3p. Cancer Cell Int 20: 248, 2020.

107. Li H, Heng B, Ouyang P, Xie X, Zhang T, Chen G, Chen Z, Cheang $\mathrm{K}$ and Lai C: Comprehensive profiling of circRNAs and the tumor suppressor function of circHIPK 3 in clear cell renal carcinoma. J Mol Histol 51: 317-327, 2020.

108. Han B, Shaolong E, Luan L, Li N and Liu X: circHIPK3 promotes clear cell renal cell carcinoma (ccRCC) cells proliferation and metastasis via altering of miR-508-3p/CXCL13 signal. Onco Targets Ther 13: 6051-6062, 2020.

109. Bray F, Ferlay J, Soerjomataram I, Siegel RL, Torre LA and Jemal A: Global cancer statistics 2018: GLOBOCAN estimates of incidence and mortality worldwide for 36 cancers in 185 countries. CA Cancer J Clin 68: 394-424, 2018

110. Qian W, Huang T and Feng W: Circular RNA HIPK3 promotes EMT of cervical cancer through sponging miR-338-3p to up-regulate HIF-1 $\alpha$. Cancer Manag Res 12: 177-187, 2020.

111. Chen S, Fan X, Gu H, Zhang L and Zhao W: Competing endogenous RNA regulatory network in papillary thyroid carcinoma. Mol Med Rep 18: 695-704, 2018.

112. Shu T, Yang L, Sun L, Lu J and Zhan X: circHIPK3 promotes thyroid cancer tumorigenesis and invasion through the Mirna-338-3p/RAB23 axis. Med Princ Pract, Oct 26, 2020 (Epub ahead of print). doi: 10.1159/000512548.

113. Ji F, Yang CQ, Li XL, Zhang LL, Yang M, Li JQ, Gao HF, Zhu T, Cheng MY, Li WP, et al: Risk of breast cancer-related death in women with a prior cancer. Aging (Albany NY) 12: 5894-5906, 2020.

114. Howell A, Anderson AS, Clarke RB, Duffy SW, Evans DG, Garcia-Closas M, Gescher AJ, Key TJ, Saxton JM and Harvie MN: Risk determination and prevention of breast cancer. Breast Cancer Res 16: 446, 2014.

115. Chen ZG, Zhao HJ, Lin L, Liu JB, Bai JZ and Wang GS: Circular RNA CirCHIPK3 promotes cell proliferation and invasion of breast cancer by sponging miR-193a/HMGB1/PI3K/AKT axis. Thorac Cancer 11: 2660-2671, 2020.

This work is licensed under a Creative Commons Attribution-NonCommercial-NoDerivatives 4.0 International (CC BY-NC-ND 4.0) License. 